\title{
Supplymentary Information
}

Switchable Dry Adhesion with Step-like Micropillars and Controllable Interfacial Contact

Yue Wang ${ }^{1 \#}$, Hongmiao Tian ${ }^{1 \#}$, Jinyou Shao ${ }^{1 *}$, Dan Sameoto ${ }^{2}$, Xiangming $\quad \mathrm{Li}^{1}$, Li Wang $^{1}$, Hong $\mathrm{Hu}^{1}$, Yucheng Ding ${ }^{1}$ and Bingheng $\mathrm{Lu}^{1}$

*Corresponding author: Jinyou Shao E-mail: jyshao@mail.xjtu.edu.cn

\# These authors contributed equally to this work

${ }^{1}$ Micro- and Nano-technology Research Center, State Key Laboratory for Manufacturing Systems Engineering, Xi'an Jiaotong University, Xi’an, Shaanxi 710049, China

${ }^{2}$ Department of Mechanical Engineering, University of Alberta, Edmonton, Alberta, T6G 1H9, Canada 
Figure S1 A typical curve of normal adhesion force versus time during preloading, dragging, and pulling. The preload is $\sim 4 \mathrm{mN}$ and the PDMS sample has an area of $\sim 4 * 4 \mathrm{~mm}^{2}$. After dragging 30um to the direction of the lower surface and returning back, the adhesion force $(\sim 67 \mathrm{mN})$ is nearly the same with that ( $\sim 1 \mathrm{mn})$ of no dragging, with small increases possibly attributed to the longer time in contact. However, if the caps are dragged 30um to the direction of the higher surface, the adhesion force $(\sim 93 \mathrm{mN})$ is much bigger. The total force isn't doubled because the internal crack or defect as observed in the videos will negate much of the benefit of the mushroom shaped structure, but if the slope or height of the step were reduced such that full contact could be made, then these fibers could show a much greater switching ratio in the future.

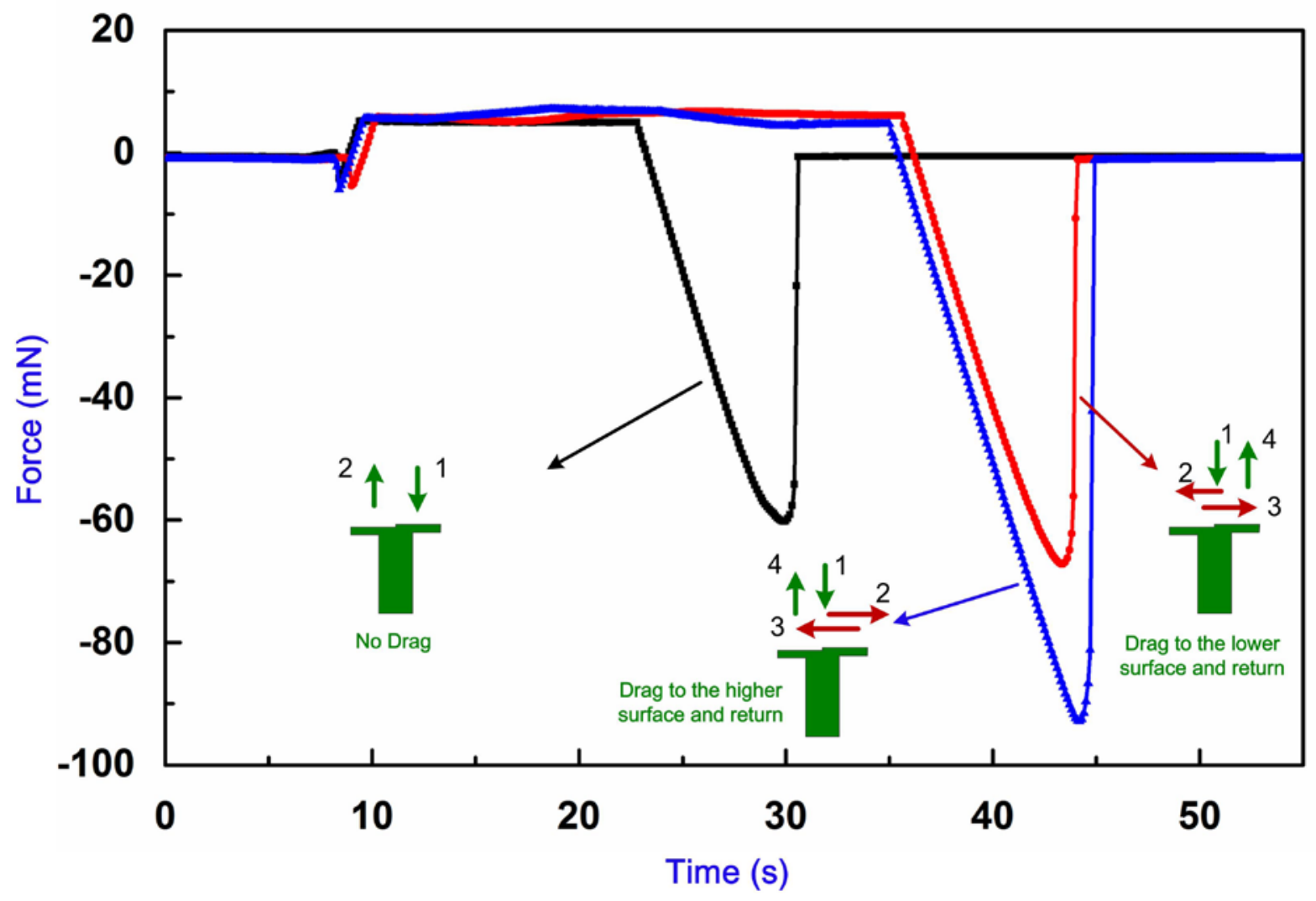


Figure S2 SEM images of micropillars array covering a large area with different step heights: $0 \mu \mathrm{m}, 0.5 \mu \mathrm{m}$ and $1.5 \mu \mathrm{m}$.
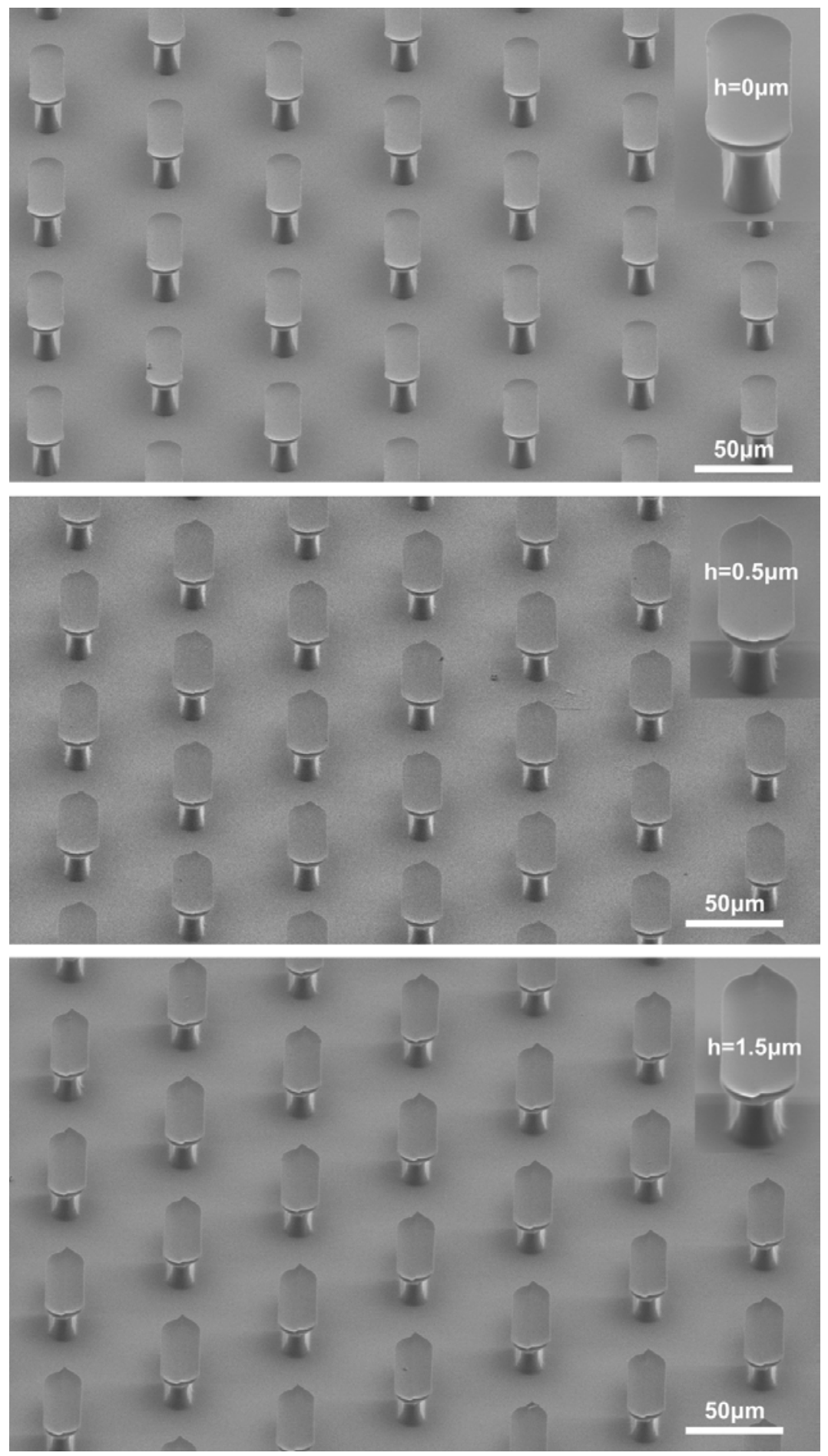

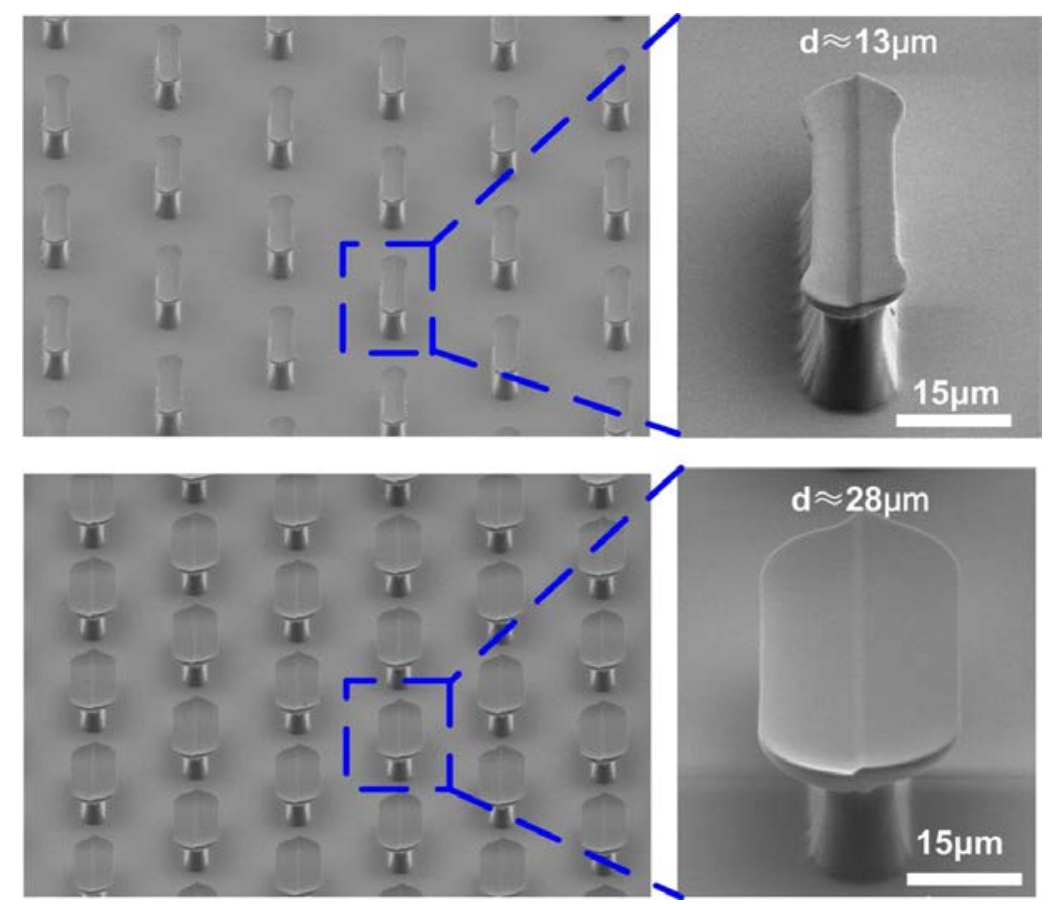

Figure S3 SEM images of micropillars array covering a large area with different cap widths:

$13 \mu \mathrm{m}$ and $28 \mu \mathrm{m}$.

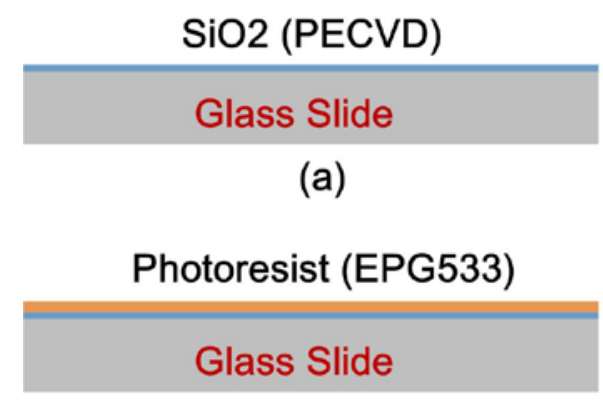

(b)

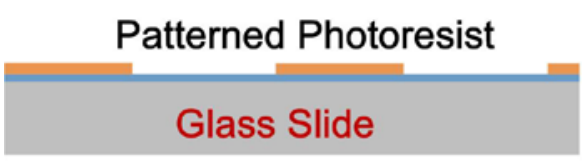

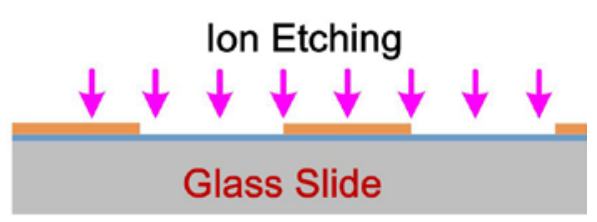

(d)

\section{Patterned $\mathrm{SiO} 2$}

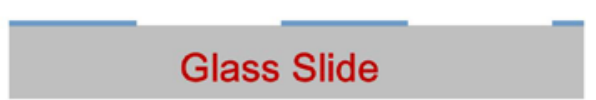

(e)

(c)

Figure S4 (a) $\mathrm{A} \mathrm{SiO}_{2}$ film is grown on the glass slide by plasma enhanced chemical vapor deposition (PECVD); (b) The photoresist (EPG533) is spin-coated on the $\mathrm{SiO}_{2}$ film; (c) The photoresist is exposed and developed; (d-e) $\mathrm{The}^{\mathrm{SiO}_{2}}$ film is patterned in an ICP-CVD chamber. 


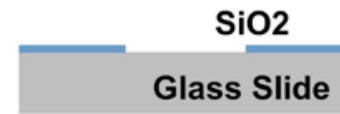

(a)

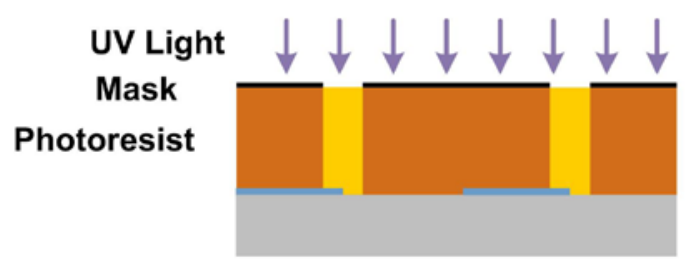

(b)

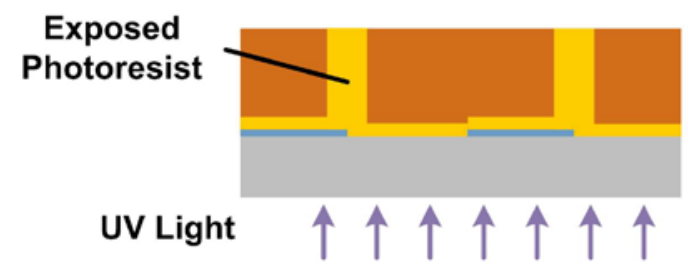

(c)

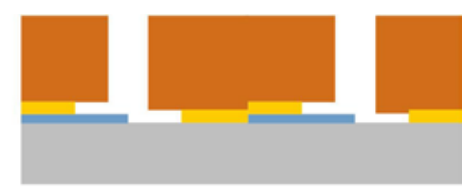

(d)

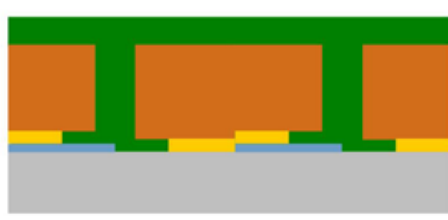

(e)

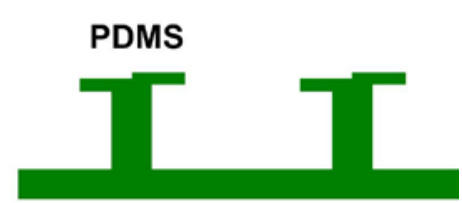

(f)

Figure S5 (a) The patterned $\mathrm{SiO}_{2}$ film; (b) The photoresist (AZ P4620) is spin-coated on the $\mathrm{SiO}_{2}$ film, followed by the exposure with a mask from the front side; (c) The AZ P4620 is exposed without any mask from the backside; (d) The AZ P4620 is precisely developed by controlling the time; (e) PDMS (Sylgard 184, Dow Chemicals) is filled into the template; (f) PDMS is demolded from the template after cured. 


\section{Video Captions}

(1) “am6b01434_si_002.avi” shows dragging 20um to the direction of the higher surface, and some of lower surfaces of the caps contact with the glass slide surface;

(2) “am6b01434_si_003.avi” shows dragging 20um to the direction of the lower surface, and the higher surfaces of the caps still contact with the glass slide surface;

(3) “am6b01434_si_004.avi” shows dragging 30um to the direction of the higher surface, and nearly all the lower surfaces of the caps contact with the glass;

(4) “am6b01434_si_005.avi” shows dragging 30um to the direction of the lower surface, and the higher surfaces of the caps begin detaching from the glass;

(5) “am6b01434_si_006.avi” shows dragging 100um to the direction of the higher surface, and the lower surfaces of the caps contact firstly with the glass. Then, both lower and higher surfaces of the caps detach from the glass surface because of large force moment. 